\title{
Study of the Performance of Various Classifiers in Labeling Non-Functional Requirements
}

\section{László Tóth}

Department of Software Engineering, University of Szeged, Hungary; e-mail: premissa@inf.u-szeged.hu

\section{László Vidács}

MTA-SZTE Research Group on Artificial Intelligence, University of Szeged, Hungary; e-mail: lac@inf.u-szeged.hu

Corresponding author: premissa@inf.u-szeged.hu

Software systems are to be developed based on expectations of the customers. These expectations are expressed using natural languages. To design software meeting the needs of the customer and the stakeholders, the intentions, feedback and reviews are to be understood accurately and without ambiguity. These textual inputs often contain inaccuracies, contradictions and are seldom given in a well-structured form. The issues mentioned in the previous thought frequently result in the program not satisfying the expectation of the stakeholders. In particular, for non-functional requirements, clients rarely emphasize these specifications as much as they might be justified. Identifying, classifying and reconciling the requirements is one of the main duty of the System Analyst, which without using a proper tool, can be very demanding and time-consuming. Tools which support text processing are expected to improve the accuracy of identification and classification of requirements even in an unstructured set of inputs. System Analysts can also use them in document archeology tasks where many documents, regulations, standards, etc. have to be processed. Methods elaborated in natural language processing and machine learning offer a solid basis. However, their usability and the possibility to improve the performance utilizing the specific knowledge from the domain of the software engineering are to be examined thoroughly. In this paper, we present the results of our work adapting natural language processing and machine learning methods for handling and transforming textual inputs of software development. The major contribution of our work is providing a comparison of the performance and applicability of the stateof-the-art techniques used in natural language processing and machine learning in software engineering. Based on the results of our experiments, tools which can support System Analysts working on textual inputs can be designed.

KEYWORDS: Requirements Engineering, Feedback processing, Natural Language Processing, Machine Learning. 


\section{Introduction}

Software systems are made based on specifications composed of functional and non-functional requirements. The needs and expectations of the customers are expressed by requirements mostly given in a natural language form. These textual documents are often ambiguous and sometimes also contain contradictions. To use the collected requirements for software design, they must be formalized and any inconsistencies need to be removed. These tasks, without using a proper tool, can be demanding and time-consuming.

Tools supporting System Analysts in requirements engineering provide templates, checklists, traceability, management and reporting environment to facilitate their work, but these tools can support processing of requirements given in textual form only to a limited extent [8].

Non-functional requirements (NFRs) are also crucial factors for software design [18]. The lack of a well-structured set of non-functional requirements can lead to an inappropriate software design and the failure of the project. Security aspects are also a critical part of the design, and their significance is growing steadily today. Every requirement related to security is to be taken into consideration, but it can be difficult without the corresponding non-functional requirements. Many NFRs are out of the analysis and those non-functional requirements considered during analysis are often weakly elaborated. Incomplete or ambiguous specifications can lead the system into an undefined state.

The quality of requirements is the primary factor of the success of a software project. Though, there are several problems to be faced with regarding the requirements. Firesmith in his article issued in Journal of Object Technology in 2007 has collected the most common issues related to requirements engineering along with some practice to solve these problems [17]. Although steps have been taken to improve the quality of the specifications, unsuccessful software projects are still being attributed largely to the inadequate requirements engineering [21].

As mentioned above, the requirements are available mainly in textual form. However, this is valid for every customer feedback, error ticket and specification. In the case of a complex project, various requirements are collected from documents, regulations reports, so
System Analyst has to process a lot of documents. These documents are well-structured and use business language. The main challenge for processing business documents is to manage texts and to identify information relevant to the application being made. In addition to the business documents, the basic sources of the requirements are the interviews with clients and stakeholders. The memos or records created during interviews are often unstructured or semi-structured and often contain ambiguities, logical fallacies. Non-functional requirements are mostly given as a part of the text but sometimes they are expressed superficially. Identifying and classifying requirements from this collection of different documents can be demanding and error-prone. Several investigations have been accomplished with remarkable results to support the identification and classification process using natural language processing and machine learning methods [1, 3, 4, 9, 24, 25, 33, 34]. Some researchers investigated the use of ontologies, which have been created based on the standards [3, 33]. Some researchers like Lu and Liang [25] or Abad et al. [1] utilized supervised learning methods, others utilized semisupervised learning techniques such as Expectation Maximization strategy [9]. Abad et al. applied also clustering techniques to identify the best method for processing requirements sentences [1]. These studies have shown that natural language processing and machine learning methods can be utilized successfully also for requirements engineering.

To apply machine learning along with natural language processing (NLP) methods, several learning examples have to be available, however, there are only a few labeled examples that can be accessed via the Internet. This is especially true for the collection of non-functional requirements which are often overlooked during the requirements elicitation. The majority of researchers have used the Tera Promise NFR dataset which was created by the students of DePaul University $[7,13]$. This dataset contains 625 examples of 15 projects classified into 12 classes. Some classes contain only very few examples which is detrimental to machine learning methods.

One possible direction for overcoming the shortage of labeled examples is using semi-supervised methods which can give better results at these conditions 
as shown by Casamayor et al. [9]. Using ontologies as a background knowledge representation is another possible solution [3, 33], but building a proper ontology-based database is a tedious and time-consuming task. Rashwan et al. have created an ontology based on the ISO/IEC 9126-1:2001. ${ }^{1}$ In addition to these strategies, corpus of requirements also can be built. Software repositories make available various open source software which can provide also sources of requirements related to the specific software [31, 32]. Extracting requirements from these repositories and building corpus can support the usage of machine learning processes effectively.

Applying NLP and machine learning methods for requirements engineering is based on the assumption that these methods can facilitate the work of System Analysts as reducing the amount of manual work, therefore, reduce the time needed for elicitation and the cost of the analysis. This assumption has also been confirmed by Groen et al. in their investigation [19]. To support System Analysts in choosing the appropriate procedure, investigations have been proceeded for comparison of the performance of the methods [1, 9, 25, 38]. The researchers found that Multinomial Naive Bayes and the Support Vector Machine (SVM) using the linear kernel had given the best performance using only a few labeled examples such as the NFR dataset.

Our work is focusing on comparison the methods including the application of the simplest deep neural network. In our former work, we have compared methods implemented in scikit-learn library [30] using the NFR dataset [38]. Our work has confirmed that the Multinomial Naive Bayes and the SVM might be the best choice if there are only a few labeled examples available. The scikit-learn library contains also a Multilayer Perceptron (MLP) classifier which has produced the worst result in our experiment owing to the small dataset. To ensure that the small dataset does not hinder the experiments, we have collected tagged posts from Stack Overflow and used them in our examinations. We have repeated the former experiments with the new dataset and also the Fully Connected Network constructed using Keras library have been tested and compared to the non-deep learning methods. The applicability and the restrictions of our methodology is shown in detail in the background section.

1 This standard has been revised and a new standard was published in 2011 as the ISO/IEC 25010:2011.
The contribution of our work is providing a comparison of the performance and applicability of the stateof-the-art techniques used in natural language processing and machine learning in software engineering. Additionally, the effects of the size of the dataset are also demonstrated.

The paper is organized as follows. The next section introduces our approach and analysis model. In Section 3 , we introduce the dataset of non-functional requirements and compare it to the dataset from Stack Overflow. Section 4 provides the findings of our analysis produced by various classifiers and compares closely related work. The related literature is briefly presented in Section 5 and we conclude the paper in Section 6.

\section{Background}

The source of the requirements is the collection of business documents, laws, regulations, and records of interviews. These documents contain texts written in natural languages. In order to be able to classify these texts, they have to be pre-processed. This transformation process converts the raw text into a vectorized form which can be used for machine learning procedures. The procedure involves common natural language techniques such as tokenization and filtering. At the end of the procedure, the input is converted into a vectorized form. The most common representation is the $t f-i d f$ model which was used also in our experiments. This representation measures the importance of a given word in a given document and produces a sparse matrix as the representation of the whole text. The measure of $t f-i d f$ is formulated as:

$t f i d f(t, d, D)=t f(t, d) * i d f(t, D)$,

where $t$ denotes terms (words in our case), $d$ denotes the document (sentences or Stack Overflow posts in our case) and $D$ denotes the collection of documents (the set of sentences or set of posts in our case). The $t f(t, d)$ is the term frequency in a given document. The $i d f$ (inverse document frequency) is formulated as:

$i d f(t, D)=\log \frac{|D|}{1+|\{d \in D: t \in d\}|}$.

For tokenization, we used the Keras Tokenizer [11] which applies filtering, lower case converting and 
splitting words before the conversion into $t f-i d f$ based vector.

Two different datasets were applied to our experiments. For the first tests, we used the Tera Promise NFR dataset which was constructed by the MS students of DePaul University [7, 13]. This dataset contains 625 requirements collected from 15 different projects. The sentences containing the requirements are classified into 12 classes from which one class represents functional requirements and the other 11 classes correspond to various types of non-functional requirements. The statistics about the classification and the related projects is shown in Table 1 and Figure 1.

Figure 1

Distribution of the Promise NFR dataset

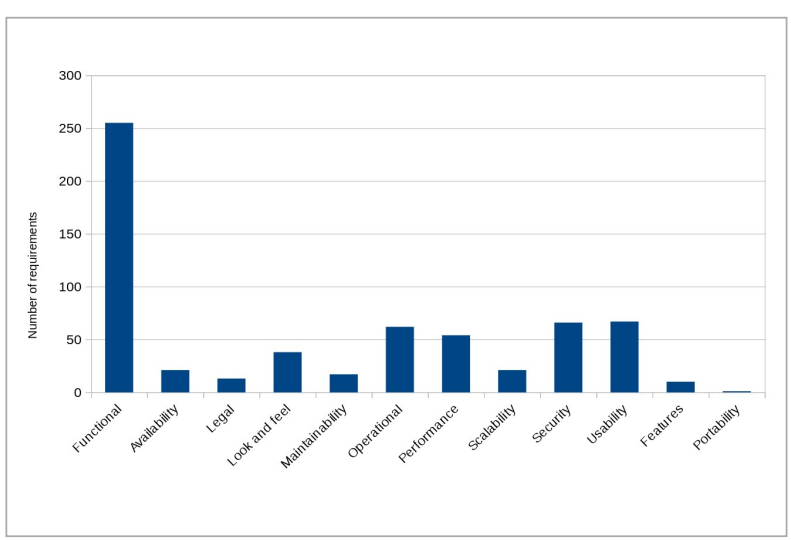

The source of the second series of our experiments was a dataset queried from Stack Overflow. The dataset is composed of a sequence of tagged posts. Posts contain English texts and sometimes code fragments are occurring. Stack Overflow is an important source also for researchers. Extracting topics related to non-functional requirements is also common $[5,6,39$, 40]. We have chosen posts tagged with performance or test related labels for our experiments. Testability and the performance are very important factors for software quality and these topics are also discussed thoroughly via Stack Overflow. This dataset contains 50000 posts where 20166 posts correspond to performance and 30753 posts correspond to test. Stack Overflow posts are multi-labeled so 919 posts are labeled both test and performance.

There were two different experiment series conducted. The first series used the Tera Promise NFR dataset and applied a set of classifiers from scikit-learn library [38]. The second series used the dataset from Stack Overflow and also applied classifiers provided by scikit-learn and supplemented by a classifier based on a simple Neural Network solution using Keras and Tensorflow [2] background.

For evaluation, we have employed precision, recall and F1 metrics. The precision is formulated as:

$$
\text { precision }=\frac{t p}{t p+f p},
$$

Table 1

Requirement labels in the 15 projects of the Promise NFR dataset

\begin{tabular}{|c|c|c|c|c|c|c|c|c|c|c|c|c|c|c|c|c|c|}
\hline \multirow{2}{*}{\multicolumn{2}{|c|}{ Requirement type }} & \multicolumn{15}{|c|}{ Project No } & \multirow{3}{*}{$\begin{array}{l}\text { Total } \\
255\end{array}$} \\
\hline & & \multirow{2}{*}{$\begin{array}{r}1 \\
20 \\
\end{array}$} & \multirow{2}{*}{$\begin{array}{r}2 \\
11 \\
\end{array}$} & \multirow{2}{*}{$\begin{array}{r}3 \\
47 \\
\end{array}$} & \multirow{2}{*}{$\begin{array}{r}4 \\
25 \\
\end{array}$} & \multirow{2}{*}{$\begin{array}{r}5 \\
36 \\
\end{array}$} & \multirow{2}{*}{$\begin{array}{r}6 \\
27 \\
\end{array}$} & \multirow{2}{*}{$\begin{array}{r}7 \\
15 \\
\end{array}$} & \multirow{2}{*}{$\begin{array}{r}8 \\
20 \\
\end{array}$} & \multirow{2}{*}{$\begin{array}{r}9 \\
16\end{array}$} & \multirow{2}{*}{$\begin{array}{c}10 \\
38\end{array}$} & \multirow{2}{*}{$\begin{array}{l}11 \\
0\end{array}$} & \multirow{2}{*}{$\begin{array}{l}12 \\
0\end{array}$} & \multirow{2}{*}{$\begin{array}{l}13 \\
0\end{array}$} & \multirow{2}{*}{$\begin{array}{l}14 \\
0\end{array}$} & \multirow{2}{*}{$\begin{array}{l}15 \\
0\end{array}$} & \\
\hline Functional & $(F)$ & & & & & & & & & & & & & & & & \\
\hline Availability & (A) & 1 & 2 & 2 & 0 & 2 & 1 & 0 & 5 & 1 & 1 & 2 & 1 & 1 & 1 & 1 & 21 \\
\hline Fault tolerance & $(\mathrm{FT})$ & 0 & 4 & 0 & 0 & 0 & 2 & 0 & 2 & 0 & 0 & 0 & 2 & 0 & 0 & 0 & 10 \\
\hline Legal & (L) & 0 & 0 & 0 & 6 & 3 & 0 & 1 & 3 & 0 & 0 & 0 & 0 & 0 & 0 & 0 & 13 \\
\hline Look and feel & $(\mathrm{LF})$ & 1 & 4 & 0 & 2 & 3 & 2 & 0 & 6 & 0 & 7 & 2 & 2 & 4 & 3 & 2 & 38 \\
\hline Maintainability & $(\mathrm{MN})$ & 0 & 0 & 0 & 0 & 0 & 4 & 0 & 2 & 1 & 0 & 1 & 3 & 2 & 2 & 2 & 17 \\
\hline Operational & (O) & 0 & 0 & 7 & 6 & 10 & 15 & 3 & 9 & 2 & 0 & 0 & 2 & 2 & 3 & 3 & 62 \\
\hline Performance & $(\mathrm{PE})$ & 2 & 6 & 2 & 2 & 4 & 1 & 2 & 17 & 4 & 4 & 3 & 5 & 0 & 1 & 1 & 54 \\
\hline Portability & $(\mathrm{PO})$ & 0 & 1 & 0 & 0 & 0 & 0 & 0 & 0 & 0 & 0 & 0 & 0 & 0 & 0 & 0 & 1 \\
\hline Scalability & (SC) & 0 & 3 & 4 & 0 & 3 & 4 & 0 & 4 & 0 & 0 & 0 & 1 & 2 & 0 & 0 & 21 \\
\hline Security & (SE) & 1 & 3 & 10 & 10 & 7 & 5 & 2 & 15 & 0 & 1 & 3 & 3 & 2 & 2 & 2 & 66 \\
\hline Usability & (US) & 3 & 6 & 8 & 4 & 5 & 13 & 0 & 10 & 0 & 2 & 2 & 3 & 6 & 4 & 1 & 67 \\
\hline Total NFRs & & 8 & 29 & 33 & 30 & 37 & 47 & 8 & 73 & 8 & 15 & 13 & 22 & 19 & 16 & 12 & 370 \\
\hline Functional & & 20 & 11 & 47 & 25 & 36 & 27 & 15 & 20 & 16 & 38 & 0 & 0 & 0 & 0 & 0 & 255 \\
\hline Total Requirements & & 28 & 40 & 80 & 55 & 73 & 74 & 23 & 93 & 24 & 53 & 13 & 22 & 19 & 16 & 12 & 625 \\
\hline
\end{tabular}


where $t p$ is the true positive which is the number of correct positive classification, $f p$ denotes the false positive which is the case when classifier accepts the example but it has to be rejected. This measure is called also as Type I error.

Recall can be formulated as:

$$
\text { recall }=\frac{t p}{t p+f n},
$$

where $f n$ denotes the false negative which is the case when classifier rejects the example but it has to be accepted. This measure is called also as Type II error.

The recall is also an important metric for our purpose because one of the most important usage of our work might be the identification of non-functional requirements from their textual context and in this case, it is crucial to find them in the input. However, the precision is as the essential metric as the recall. Taking into account the purpose of the classifier of non-functional requirements the most adequate metric is the $\mathrm{F} 1$.

$\mathrm{F} 1$ is formulated as:

$$
F 1=2 * \frac{\text { precision } * \text { recall }}{\text { precision }+ \text { recall }}
$$

Note: $\mathrm{F} 1$ is a special case of $\mathrm{F}$-measure with $\beta=1$ value. The F-measure is formulated as:

$$
F_{\beta}=\left(1+\beta^{2}\right) * \frac{\text { precision } * \text { recall }}{\beta^{2} * \text { precision }+ \text { recall }} .
$$

During the Stack Overflow based experiments, the calculation of the metrics has been performed using classification reports provided by the scikit-learn library. The report uses the calculation methods which were applied directly in Promise based experiments. For this former dataset, the averaged measures were calculated manually but, for dataset obtained from Stack Overflow, the average values were produced by the classification report. Four types of averages were calculated by the report which are the micro, macro, weighted and samples averages.

Let denote $y_{l}$ the set of label $l$ predicted by a classifier and $y_{l}{ }_{l}$ the set of classes with true label $l$. Let $y=U y_{l}$ and $y^{\wedge}=U y_{l}$, where $l \in L$ and $L$ is the set of labels. Then the micro average of precision can be given as

$$
P(y, \hat{y})=\frac{|y \cap \hat{y}|}{|y|} .
$$

The same is done for recall:

$$
R(y, \hat{y})=\frac{|y \cap \hat{y}|}{|\hat{y}|} .
$$

For macro averages, the formulas are the following:

$$
\begin{aligned}
& P(y, \hat{y})_{\text {macro }}=\frac{1}{L} \sum_{l \in L} P\left(y_{l}, \hat{y}_{l}\right) \\
& R(y, \hat{y})_{\text {macro }}=\frac{1}{L} \sum_{l \in L} R\left(y_{l}, \hat{y_{l}}\right) .
\end{aligned}
$$

Similar formulas can be used for sample averages, where $S$ denotes the set of samples:

$$
\begin{aligned}
& P(y, \hat{y})_{\text {samples }}=\frac{1}{S} \sum_{s \in S} P\left(y_{s}, \hat{y}_{s}\right) \\
& R(y, \hat{y})_{\text {samples }}=\frac{1}{S} \sum_{s \in S} R\left(y_{s}, \hat{y}_{s}\right) .
\end{aligned}
$$

For weigted averages, the following formulas can be applied:

$$
\begin{aligned}
& P(y, \hat{y})_{\text {weighted }}=\frac{1}{\sum_{l \in L}\left|\hat{y}_{l}\right|} \sum_{l \in L}\left|\hat{y}_{l}\right| P\left(y_{l}, \hat{y}_{l}\right) \\
& R(y, \hat{y})_{\text {weighted }}=\frac{1}{\sum_{l \in L}\left|\hat{y}_{l}\right|} \sum_{l \in L}\left|\hat{y_{l}}\right| R\left(y_{l}, \hat{y}_{l}\right) .
\end{aligned}
$$

\section{Experiments}

As we have mentioned in the previous section, two series of experiments were performed with two different datasets. Before the experiments datasets were preprocessed. During this transformation filtering and tokenization were applied and the resulted text was transformed into $t f-i d f$ representation.

The Promise NFR dataset contains only one label for each example but the dataset extracted from Stack Overflow is multilabeled. We selected only two labels (performance and test) for extraction to obtain 
a well-balanced input. During the preprocessing the labels of dataset from Stack Overflow were also transformed using one-hot encoding and the labels different from our interest were filtered out.

In the first experiments, we accomplished classification processes using algorithms implemented in scikit-learn library on the dataset of Promise NFR and compared the results each other and the results obtained by Cassamayor et al. in their experiments [9]. The objective of these experiments was to determine the best algorithm of classification implemented in scikit-learn for requirements classification tasks considering precision, recall and F-measure, complemented by execution time. For these first series of experiments, we applied the following classifiers: Multinomial-, Gaussian- and Bernoulli Naive Bayes, Support Vector Machine with linear kernel, Linear Logistic Regression, Label Propagation, Label Spreading, Decision Tree, Extra Tree, K-Nearest Neighbour and Multi Layer Perceptron. The selection process was also influenced by the resource requirements of the classifiers.

The experiments were executed using repeated K-Fold cross-validation method. Both the number of groups and the repetition number were set to 10 . The classification was performed using one-versus-rest strategy for each class. Using this strategy, the examined class is fitted against the other classes. Precision, recall and F-measure were calculated for each test and each class by the corresponding scikit-learn method and the results were averaged for each classifier. Averaged variance was calculated as well and also the F-measure value was computed for every classifier using the averaged precision and recall because the averaged F-measure does not hold any useful information using this method of calculation.

The Stack Overflow based experiments applied Multinomial, Gaussian- and Bernoulli Naive Bayes, Support Vector Machine with linear kernel, Linear Logistic Regression, Decision Tree, Extra Tree, and K-Nearest Neighbour classifiers from scikit-learn library, and the experiments were supplemented using a fully connected neural network with Keras and Tensorflow back-end. The MLP test was removed from this series because the implemented fully connected network gives better scalability and the applied strategy is similar in the two cases. The LabelPropagation and the LabelSpreading were also removed because we experienced performance issues applying it on the Stack Overflow dataset.

The original dataset extracted from Stack Overflow contains 50000 examples which were split to a trainand a test-set. The train-set contains 35000 trainposts whereas the test-set contains 15000 posts.

The classifiers from scikit-learn were applied using one-versus-rest strategy. The precision, recall and the F1-measure were calculated for each class and the averages were also determined as we described it in the previous section.

The Fully Connected Network was constructed using Keras libraries and Tensorflow back-end. Our model contains 1024 units in the first layer. The number of units and the input shape can be varied, the shape has to be aligned with the dimension of $t f-i d f$ vectors. In our case, this dimension is 3000 which is the number of the selected most frequent words from the input dataset. As mentioned before, this hyperparameter can be varied, however, our experiments did not yield better results in case of the bigger dimension. In general, this parameter depends on the size of the vocabulary of the dataset in question.

For the purpose of the regularization, the dropout technique was applied. The dropout regularization selects a given proportion of the units randomly and inactivates them during the learning phase. The ratio of the neurons is to be inactivated is a hyperparameter which can be tuned during the experiments. The activation function of the first layer is the ReLU (Rectified Linear Unit). This function produces 0 for every negative input and any positive $\mathrm{x}$ it returns with that value. (Formula: $f(x)=\max (0, x)$ ). The second layer is a softmax layer whose output size is the same as the number of classes under investigation. The softmax function can be written as:

$\sigma(z)_{j}=\frac{e^{z_{j}}}{\sum_{k=1}^{K} e^{z_{k}}}$,

where $K$ is the number of classes. The formula provides a probability value for every class used in the model.

The network was trained using the categorical cross-entropy as a loss function and the adam optimizer [23] was applied. The equiation of categorical cross-entropy is: 
$-\frac{1}{N} \sum_{i=1}^{N} \sum_{c=1}^{C} \mathbf{1}_{y_{i} \in C_{c}} \log p_{\text {model }}\left[y_{i} \in C_{c}\right]$,

Adam (Adaptive Moment Estimation) is a gradient-based optimizer for stochastic objective functions which applies adaptive estimation of lower-order moments which help the algorithm to not stuck in a local optimum. The optimizer combines the advantages of AdaGrad and RMSProp methods [23]. The update rule of Adam is:

$$
\theta_{t+1}=\theta_{t}-\frac{\eta}{\sqrt{\hat{v}_{t}}+\epsilon} \hat{m}_{t}
$$

where $\hat{m}_{t}$ and $\hat{v}_{t}$ are the second order moments which are calculated as:

$$
\begin{aligned}
& \hat{m}_{t}=\frac{m_{t}}{1-\beta_{1}^{t}} \\
& \hat{v}_{t}=\frac{v_{t}}{1-\beta_{2}^{t}},
\end{aligned}
$$

where $m_{t}$ and $v_{t}$ are the first-order moments which are updated as:

$m_{t}=\beta_{1} m_{t-1}+\left(1-\beta_{1}\right) g_{t} v_{t}=\beta_{2} v_{t-1}+\left(1-\beta_{2}\right) g_{t}^{2}$.
For $\beta_{1}, 0.9$ is proposed by the authors as the default value, whereas, for $\beta_{2}, 0.999$ and $10^{-8}$ for [23]. We used the default values for our experiments.

In addition to the performance of classifiers, the execution time was also measured during the experiments.

\section{Results}

In Table 2, the measured averages of Precision, Recall and F-measure related to the first series of our experiments based on the Tera Promise NFR dataset with their averaged variance are presented. As mentioned in the previous section, averaging $\mathrm{F}$-measure does not hold valuable information so F-measure was computed based on averages precision and recall. The averages of precision, recall and F-measure values and their variance are illustrated in Figure 2. For comparison purpose, the results are illustrated using line diagrams. The results show that the SVM has produced the best precision value, however, the recall is only $65 \%$ which is the median value of the results. The Multinomial Naive Bayes and Logistic Regression have produced the best F1 values but the F1 value of the SVM is only $1 \%$ lower. As one can see in the chart, the MLP has produced the worst result. The size of

\begin{tabular}{|c|c|c|c|c|c|c|c|}
\hline \multirow{2}{*}{ Classifier } & \multicolumn{3}{|c|}{ Average } & \multirow{2}{*}{$\begin{array}{c}\text { Comp } \\
\text { F }\end{array}$} & \multicolumn{3}{|c|}{ Variance } \\
\hline & $P$ & $\mathrm{R}$ & $\mathrm{F}$ & & $\mathrm{P}$ & $\mathrm{R}$ & $\mathrm{F}$ \\
\hline BernoulliNB & 0.43 & 0.22 & 0.25 & 0.29 & 0.18 & 0.09 & 0.06 \\
\hline $\mathrm{DT}$ & 0.66 & 0.64 & 0.62 & 0.65 & 0.01 & 0.03 & 0.01 \\
\hline $\mathrm{ET}$ & 0.63 & 0.62 & 0.59 & 0.62 & 0.01 & 0.04 & 0.02 \\
\hline ETs & 0.63 & 0.63 & 0.59 & 0.63 & 0.01 & 0.04 & 0.02 \\
\hline GNB & 0.72 & 0.69 & 0.67 & 0.70 & 0.02 & 0.02 & 0.02 \\
\hline KNeighbours & 0.71 & 0.52 & 0.55 & 0.60 & 0.08 & 0.07 & 0.05 \\
\hline LabelPropagation & 0.70 & 0.68 & 0.65 & 0.69 & 0.02 & 0.03 & 0.02 \\
\hline LabelSpread & 0.70 & 0.67 & 0.65 & 0.68 & 0.02 & 0.03 & 0.02 \\
\hline Logistic & 0.87 & 0.67 & 0.72 & 0.76 & 0.01 & 0.05 & 0.03 \\
\hline$\underline{\text { MLP }}$ & 0.38 & 0.66 & 0.36 & 0.48 & 0.01 & 0.03 & 0.01 \\
\hline MultinomialNB & 0.84 & 0.68 & 0.72 & 0.75 & 0.02 & 0.03 & 0.02 \\
\hline SVM & 0.89 & 0.65 & 0.71 & 0.75 & 0.01 & 0.05 & 0.02 \\
\hline
\end{tabular}

Table 2

Precision, recall and F-measure values during the classification of the Promise NFR 
Figure 2

Precision, recall, f-measures (left) and their variance (right) during classification of the Promise NFR

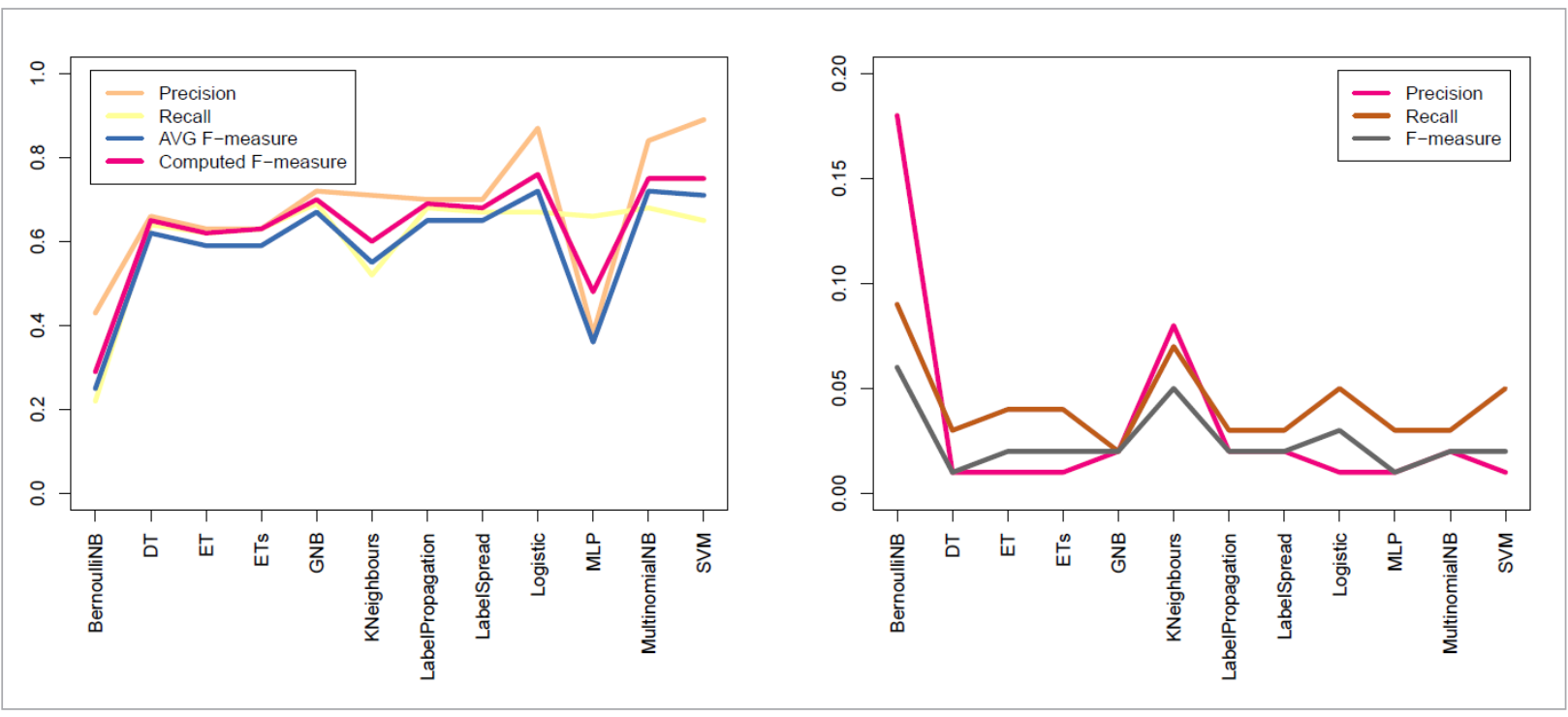

the dataset is too small for applying the Multilayer Perceptron classifier, but this result can be explained with the underfitted model.

The variance of the measures can be seen on the right side of the chart. As one can see the Bernoulli Naive Bayes and the KNeighbours have resulted in the largest variance, but the top 3 classifiers (Logistic Regression, Multinomial Naive Bayes and SVM) have a small variance. The average variance is relatively low, the values are lower than $5 \%$ for every metrics.

As the results of our first-series experiments present, regarding the precision the Multinomial Naive Bayes Classifier, Support Vector Machine with linear kernel and Linear Logistic Regression have produced the best values. The Naive Bayes Classifier was found also in former researches as the best classifier for classification of requirement sentences comparing it to other classifiers such as the tf-idf classifier [9], the k-Nearest Neighbour [1, 9], the Bittern Topic Model (BTM) or the Latent Dirichlet Allocation (LDA) [1]. $\mathrm{Lu}$ and Liang have found that the SVM classifier has performed best during their research [25].

The second series of the experiments used dataset obtained from Stack Overflow. This dataset is large enough for machine learning purpose. While the Promise NFR contains 625 examples, this dataset contains 50000 examples. The main drawback re- garding Stack Overflow dataset is its noisy nature. Noisiness means in our case that there are several false or off-topic posts presents in the database. Noisiness also means that there are many code-fragments presented which have to be also filtered out.

The results of the second series of experiments (Stack Overflow) are presented in Table 3 and Table 4, re-

\section{Table 3}

Precision, recall and F-measure values during the classification of the dataset from Stack Overflow

\begin{tabular}{|c|c|c|c|c|c|c|}
\hline \multirow{2}{*}{ Classifier } & \multicolumn{4}{|c|}{$\begin{array}{l}\text { micro } \\
\text { average }\end{array}$} & \multicolumn{2}{|c|}{$\begin{array}{l}\text { macro } \\
\text { average }\end{array}$} \\
\hline & $\mathrm{P}$ & $\mathrm{R}$ & $\mathrm{F}$ & $\mathrm{P}$ & $\mathrm{R}$ & $\mathrm{F}$ \\
\hline BernoulliNB & 0.91 & 0.91 & 0.91 & 0.90 & 0.90 & 0.90 \\
\hline GaussianNB & 0.82 & 0.81 & 0.81 & 0.84 & 0.84 & 0.81 \\
\hline MultinomialNB & 0.92 & 0.91 & 0.92 & 0.92 & 0.92 & 0.92 \\
\hline DecisionTree & 0.91 & 0.91 & 0.91 & 0.91 & 0.90 & 0.90 \\
\hline ExtraTree & 0.84 & 0.84 & 0.84 & 0.83 & 0.83 & 0.83 \\
\hline LogisticRegr & 0.95 & 0.95 & 0.95 & 0.95 & .95 & 0.95 \\
\hline KNeighbours & 0.80 & 0.80 & 0.80 & 0.82 & 0.76 & 0.77 \\
\hline SVM & 0.95 & 0.94 & 0.94 & 0.94 & 0.94 & 0.94 \\
\hline FullyConnected & 0.96 & 0.94 & 0.95 & 0.96 & 0.94 & 0.95 \\
\hline
\end{tabular}


Table 4

Precision, recall and F-measure values during the classification of the dataset from Stack Overflow

\begin{tabular}{l|c|c|c|c|c|c|}
\hline \multirow{2}{*}{\multicolumn{1}{c|}{ Classifier }} & \multicolumn{5}{|c}{$\begin{array}{c}\text { weighted } \\
\text { average }\end{array}$} \\
\cline { 2 - 7 } & P & R & F & P & R & F \\
\hline BernoulliNB & 0.91 & 0.91 & 0.91 & 0.91 & 0.91 & 0.91 \\
\hline GaussianNB & 0.87 & 0.81 & 0.81 & 0.82 & 0.82 & 0.81 \\
\hline MultinomialNB & 0.92 & 0.91 & 0.92 & 0.93 & 0.92 & 0.92 \\
\hline DecisionTree & 0.91 & 0.91 & 0.91 & 0.90 & 0.91 & 0.90 \\
\hline ExtraTree & 0.84 & 0.84 & 0.84 & 0.80 & 0.85 & 0.80 \\
\hline LogisticRegr & 0.95 & 0.95 & 0.95 & 0.95 & 0.96 & 0.95 \\
\hline KNeighbours & 0.80 & 0.80 & 0.79 & 0.80 & 0.80 & 0.80 \\
\hline SVM & 0.95 & 0.95 & 0.94 & 0.94 & 0.95 & 0.94 \\
\hline FullyConnected & 0.96 & 0.94 & 0.95 & 0.96 & 0.95 & 0.95
\end{tabular}

\section{Figure 3}

Average precision, recall values during classification of the dataset from Stack Overflow

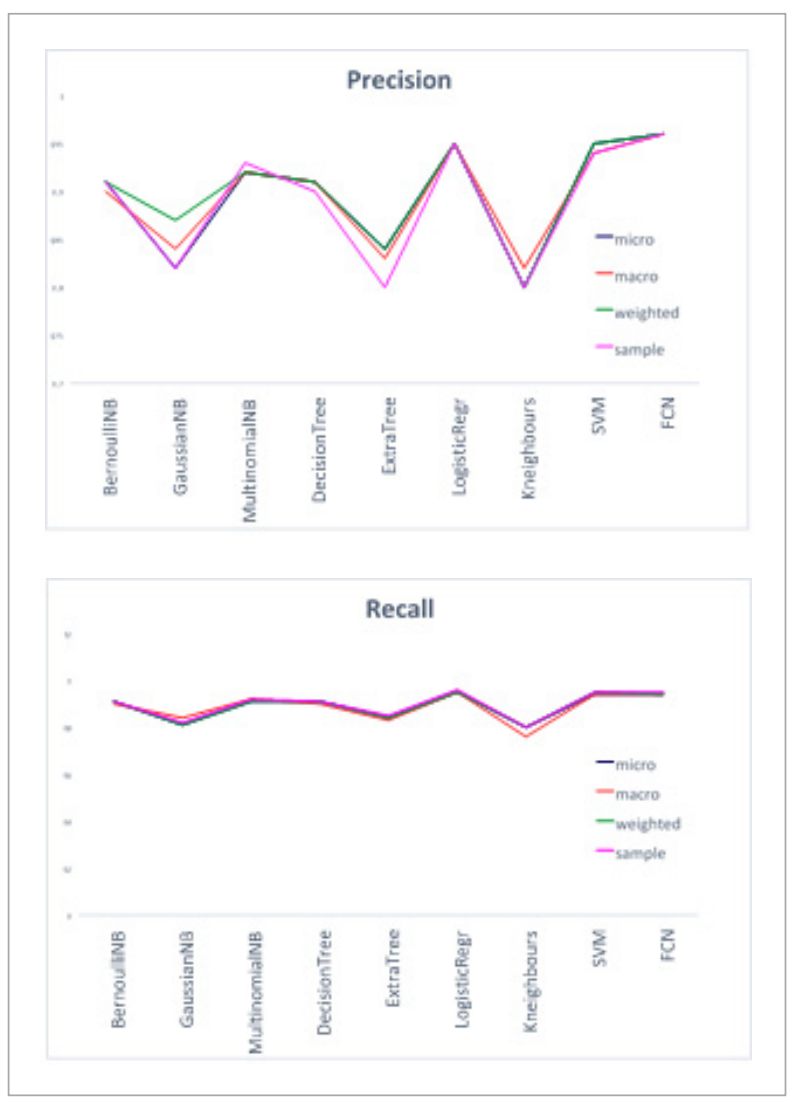

spectively. The results are also illustrated in Figure 3 and in Figure 4. According to the results of the Logistic Regression, the SVM and the FCN have produced the best values. The FCN has yielded the strongest values of precision and F-measure in all calculated averages. However, regarding the F-measure the results of Logistic Regression are the same as those of the FCN. In the case of the recall, the Logistic Regression has outperformed the other classifiers, however, the results of the FCN are only $0.1 \%$ lower.

\section{Figure 4}

Average F1-measure during the classification of the dataset from Stack Overflow

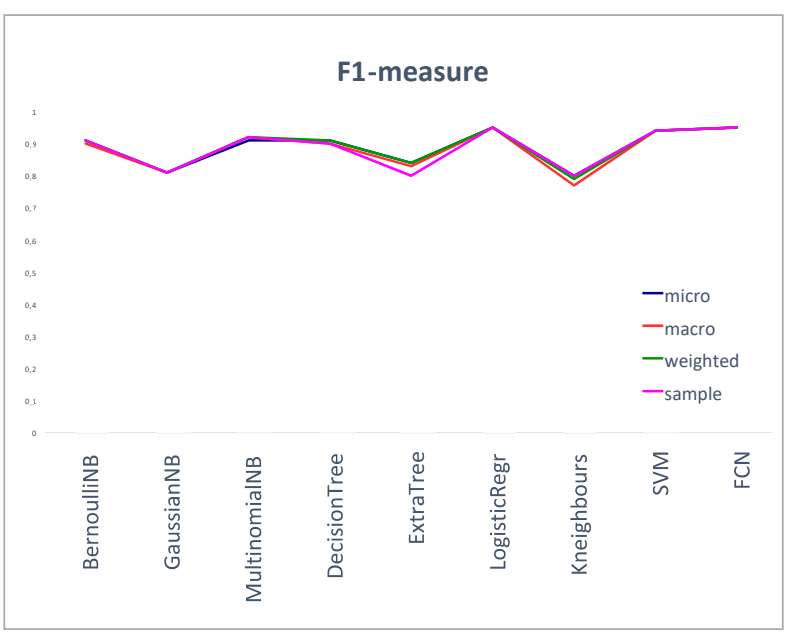

The averages were calculated using the micro, macro, weighted and samples calculation method. The formulas of these methods are presented in the Section 2 of this paper. The suitability of these methods depends on the input. Macro averages take the values calculated for every class and calculate their average. In the case of a well-balanced dataset, this average can be useful, but when the size of the classes varies, this method is not recommended. The micro average can be applied for any cases and it is the best choice if the size of the classes varies. If the size of the classes is also an important factor, weighted averages can be proposed. Sample averages calculate the averages of the values of the instances. As one can see in Figures 3 and 4 , these values differ but can be used in a similar way to characterize classifiers.

The significance of the difference was tested using the first five classifiers, the FCN, the SVM, the Logistic Regression, the Multinomial Naive Bayes and 
the Decision Tree Classifier. For the significance test, we have applied one-way repeated measures ANOVA test. The $p$-value of the test is 0.000575 , which is significant at $p<0.05$. Because the assumptions of ANOVA as the samples are drawn from a normal distribution and the variance homogeneity is not held, we also performed a repeated measures Friedman Test [14]. The $p$-value in this case is 0.10739 which is not significant at $\mathrm{p}<0.05$. As mentioned before, the value of the F-measure is the same of the FCN and the Logistic Regression.

The variance was also calculated, however, only two classes were included in the classification. The results of the calculation are shown in Table 5 and in Figure 5. The results show that Gaussian Naive Bayes has produced the biggest variance regarding the precision whereas the KNN yielded the biggest value regarding to the recall. Those classifiers, which presented the best values, produced also a small variance.

The results of the second series of the experiments (Stack Overflow based) confirmed the result of the first series (Tera Promise based) as the SVM and the Logistic Regression have produced the best values using scikit-learn classifiers. However, Multinomial Naive Bayes has also given good results, its performance appeared to be a bit worse than the results of the top three. These values suggest that the linear classifiers can be applied for classifying, labeling or tagging requirements given in natural language form. The result of the FCN shows that if there are enough examples

Table 5

Variance statistics during the classification of the dataset from Stack Overflow

\begin{tabular}{l|c|c|c}
\multicolumn{1}{c|}{ Classifier } & \multicolumn{3}{|c}{ Variance } \\
\cline { 2 - 4 } BernoulliNB & $\mathrm{P}$ & $\mathrm{R}$ & $\mathrm{F}$ \\
\hline GaussianNB & 0.045 & 0.045 & 0.045 \\
\hline MultinomialNB & 3.200 & 3.645 & 0.005 \\
\hline DecisionTree & 0.320 & 0.020 & 0.045 \\
\hline ExtraTree & 0.4045 & 0.080 & 0.045 \\
\hline LogisticRegr & 0.020 & 0.500 & 0.405 \\
\hline KNeighbours & 0.320 & 6.480 & 1.125 \\
\hline SVM & 0.045 & 0.320 & 0.320 \\
\hline FullyConnected & 0.020 & 0.020 & 0.020
\end{tabular}

\section{Figure 5}

Variance statistics during the classification of the dataset from Stack Overflow

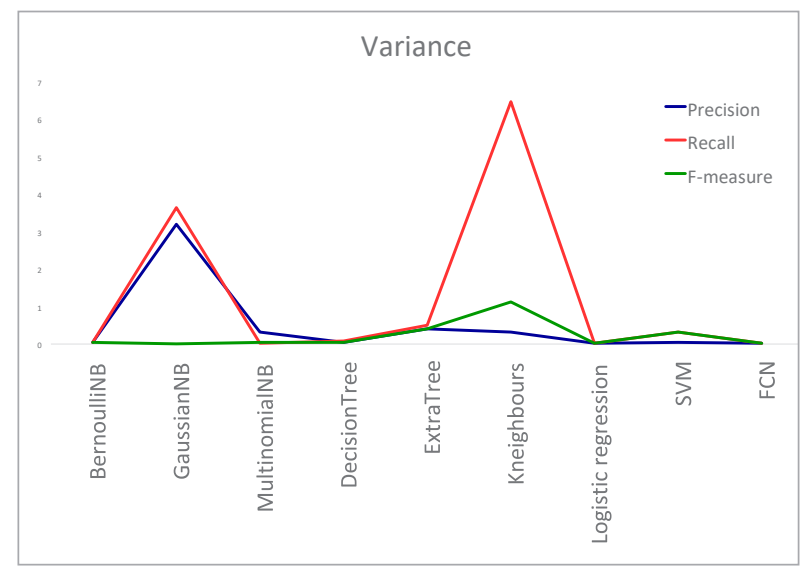

available, the neural network models can outperform the classic models of machine learning.

During the FCN test, we applied a simple fully connected network with the dropout regularization and the Adam optimizer. The hyperparameters such as the number of neurons, the dropout keep ratio, the batch size or the number of epochs were also adjusted, and the results were checked. The best results were achieved using 1024 neurons in the first layer, the 0.1 value for dropout parameter and 100 for batch size. The training phase contained 3 epochs. The maximum difference between the worst and the best results was only $0.2 \%$ during the parameter changes.

As mentioned in the previous sections, the execution time was also measured for every classifier during both series of the experiments. Execution time is also an important factor for practice which can help System Analyst to choose the appropriate tool for requirement elicitation. The values of the execution time of the second series of our experiments are presented in Table 6. The two series of the experiments were performed in a different hardware context, therefore, the results cannot be compared. The second series, however, provides useful information about the expectable execution time using a standard desktop environment with Intel I7-3770 3.9 GHz CPU and 8 GB RAM with 240 GB SSD. The operating system is Windows 1064 bit.

The execution times are given in seconds. However, the actual execution time depends on the dataset under in- 
Table 6

Execution time of the learning process using the dataset from Stack Overflow

\begin{tabular}{l|c}
\hline BernoulliNB & 3.80 \\
\hline GaussianNB & 6.67 \\
\hline MultinomialNB & 0.99 \\
\hline DecisionTree & 162.26 \\
\hline ExtraTree & 3.33 \\
\hline LogisticRegression & 11.18 \\
\hline KNeighbours & 4290.83 \\
\hline SVM & 10.91 \\
\hline FCN & 50.92 \\
\hline
\end{tabular}

vestigation, these results can give a hint for the choice in case the running time is an important factor. The best result has been presented using Multinomial Naive Bayes. This classifier was also the winner regarding the execution time also in our first experiments. The worst result has been produced by KNeighbours classifier. This epoch lasted 4290.83 seconds which is very high comparing to other classifiers. The execution time of the FCN is also a reasonable result which implies that it is worth to move to the area of the Deep Learning also in classification non-functional requirements.

\section{Related Work}

The problem of processing requirements documents using natural language processing and machine learning methods has been a research topic for decades [4]. Although non-functional requirements are less dependent on the application domain, it is not a trivial problem to set up a general list of NFR types. The types identified in the literature are widespread, for example, Chung et al. [12] identified 156 NFR categories, while Mairiza et al. [28] separated 114 different NFR classes in their work, on the contrary to the 6 high-level categories defined by the ISO/IEC 25010:2011 standard.

A fundamental study of NFR classification is published relatively lately in 2006 by Cleland-Huang et al. [13]. They used 14 NFR categories separated from functional requirements. More than 600 requirements from 15 projects were collected and manually categorized to train and test their categorization methods. Cleland-Huang et al. achieved high recall with the tradeoff of really low precision. This experiment was reproduced by several researchers in the past [10, 37]. Casamayor et al. [10] employed multinomial Naive Bayes classifier coupled with an Expectation Maximization algorithm.

Requirements traceability is a related field, where NLP and information retrieval techniques are frequently applied [26, 27, 41]. Hindle et al. [20] used topic modeling to link NFRs to topics found in commit messages. Falessi et al. [16] conducted a largescale experiment with various NLP techniques including different algebraic models, term weightings and similarity metrics in order to detect identical nonfunctional requirements.

Sharma et al. [36] addressed the NFR extraction problem with a rule-based approach. They implemented a framework for NFR analysis including a DSL language. Sawyer et al. have focused on document archaeology and created an NLP based tool called REVERE to support business analysts in the investigation of different documents containing requirements [35]. This tool has utilized some standard NLP techniques like part-of-speech tagging or semantic tagging and determination of modality.

Denger et al. examined the ambiguity of requirements sentences and investigated the use of language patterns for rewriting these requirements into less ambiguous sentences [15]. The ambiguity of requirements is one of the most prominent issues in requirements engineering which has to be resolved as pointed by Firesmith in his paper [17]. Ambiguity, completeness, conformity of requirements were examined and discussed also by Kang and Park using linguistics viewpoint [22]. The researchers developed a requirement-grammar and also applied error patterns for developing a tool for parsing the manually annotated corpus built by system-engineering related texts. The annotated corpus can be then used for training error model which can be applied to check the exactness of the requirements.

From the perspective of mining software repositories, Paixao et al. [29] investigated the relationship between built results obtained from continuous integration tools with non-functional requirements.

To overcome the scarcity of the labeled examples available via the Internet, extracting requirements re- 
lated information from social networks has become a frequently examined area. Portugal and do Prado Leite [31] applied the pattern-search method and extracted requirements related information from GitHub. They have also built a corpus of requirements based on the information retrieved from README files located on GitHub repository [32]. Stack Overflow is also a popular repository among the researchers. Zou et al. examined the repository via utilizing the Latent Dirichlet Allocation (LDA) to discover the main topics of discussion. [39]. These topics are related to various NFR which were also identified by the researchers. The topics which are the focus of developers was investigated also by Barua et al. [6] using LDA on Stack Overflow textual database.

\section{Conclusions}

Identifying and classifying non-functional requirements is a crucial duty of System Analysts which can be a demanding and error-prone task without using a proper tool. The collection of the requirements expressed using natural language form are originated from customers and stakeholders. Natural Language Processing techniques along with Machine Learning can support the processing of the requirements given in the textual form.

In this paper, we have demonstrated the usability of various machine learning methods along with the transformation of the textual input into an appropriate form which is processable by implemented ma-

\section{References}

1. Abad, Z. S. H, Karras, O., Ghazi, P., Glinz, M., Ruhe, G., Schneider, K. What Works Better? A Study of Classifying Requirements. Proceedings 2017 IEEE 25th International Requirements Engineering Conference, RE 2017, 2017, 496-501. https://doi.org/10.1109/RE.2017.36

2. Abadi, M., Agarwal, A., Barham, P., Brevdo, E., Chen, Z., Citro, C., Corrado, G. S., Davis, A., Dean, J., Devin, M., Ghemawat, S., Goodfellow, I., Harp, A., Irving, G., Isard, M., Jia, Y., Jozefowicz, R., Kaiser, L., Kudlur, M., Levenberg, J., Mané, D., Monga, R., Moore, S., Murray, D., Olah, C., Schuster, M., Shlens, J., Steiner, B., Sutskever, I., Tal- chine learning algorithms. The methods have been compared with each other using precision, recall and F1 metrics and also a significance test has been performed. Two series of experiments were executed. We used the small-sized Tera Promise NFR dataset to the first series and the large dataset extracted from Stack Overflow for the second series of experiments. The results are presented in the results section.

The results of our experiments show that the linear classification algorithm produced the best values using both of the small and the large datasets. The winners are Multinomial Naive Bayes, Support Vector Machine and the Logistic Regression but Decision Tree also produced good results. Using the dataset from Stack Overflow, the Fully Connected Network produced the best values and outperformed the other classifiers.

The performance metrics along with execution times present that using NLP and machine learning techniques provides a reasonable solution for the base technology of tools supporting System Analysts in requirement elicitation process. The precision and recall can be both above the $95 \%$ which is a good starting point for practical usage, however, these values are to be improved.

Our future research plans are to continue studying neural networks by involving deep networks and recurrent networks. Examinations will be extended using another representation of inputs such as embedding techniques which can represent also the contextual information of a textual element and also some grammatical features can be also captured.

war, K., Tucker, P., Vanhoucke, V., Vasudevan, V., Viégas, F., Vinyals, O., Warden, P., Wattenberg, M., Wicke, M., Yu, Y., Zheng, X. TensorFlow: Large-Scale Machine Learning on Heterogeneous Systems, 2015.

3. Al Balushi, T. H., Sampaio, P. R. F., Dabhi, D., Loucopoulos, P. ElicitO: A Quality Ontology-Guided NFR Elicitation Tool. Requirements Engineering: Foutndation for Software Quality, 2007, 306319. https://doi. org/10.1007/978-3-540-73031623.

4. Ambriola, V., Gervasi, V. Processing Natural Language Requirements. Proceedings 12th IEEE International 
Conference Automated Software Engineering, 1997, 36-45.

5. An, L., Mlouki, O., Khomh, F., Antoniol, G. Stack Overflow: A Code Laundering Platform? SANER 2017 - 24th IEEE International Conference on Software Analysis, Evolution, and Reengineering, 2017, 283-293. https:// doi.org/10.1109/SANER.2017.7884629

6. Barua, A., Thomas, S. W., Hassan, A. E. What Are Developers Talking About? An Analysis of Topics and Trends in Stack Overflow. Empirical Software Engineering, 2014, 19(3), 619-654. https://doi.org/10.1007/s10664012-9231-y

7. Caglayan, B., Kocaguneli, E., Krall, J., Peters, F., Turhan, B. The PROMISE Repository of Empirical Software Engineering Data, 2012.

8. Carrillo de Gea, J. M., Nicolás, J., Alemán, J. L. F., Toval, A., Ebert, C., Vizcaíno, A. Requirements Engineering Tools. IEEE Software, 2011, 28(4), 86-91. https://oi. org/10.1109/MS.2011.81

9. Casamayor, A., Godoy, D., Campo, M. Identification of NonFunctional Requirements in Textual Specifications: A Semi-supervised Learning Approach. Information and Software Technology, 2010, 52(4), 436-445. https://doi.org/10.1016/j.infsof.2009.10.010

10. Casamayor, A., Godoy, D., Campo, M. Functional Grouping of Natural Language Requirements for Assistance in Architectural Software Design. Knowledge-Based Systems, 2012, 30, 78-86. https://doi.org/10.1016/j.knosys.2011.12.009

11. Chollet, F. K., 2015. https:// github.com/fchollet/keras.

12. Chung, L., Nixon, B. A., Yu, E., Mylopoulos, J. Non-Functional Requirements in Software Engineering, 2000. https://doi.org/10.1007/978-1-4615-5269-7

13. Cleland-Huang, J., Settimi, R., Zou, X., Solc, P. Automated Classification of Non-Functional Requirements. Requirements Engineering, 2007, 12(2), 103-120. https:// doi.org/10.1007/s00766-007-0045-1

14. Demšar, J. Statistical Comparisons of Classifiers over Multiple Data Sets, 2006. Technical report, 2006.

15. Denger, C., Berry, D. M., Kamsties, E. Higher Quality Requirements Specifications through Natural Language Patterns. Proceedings of the IEEE International Conference on Software - Science, Technology \& Engineering (SwSTE’03), 2003, 1-11.

16. Falessi, U., Cantone, G., Canfora, G. A Comprehensive Characterization of NLP Techniques for Identifying Equivalent Requirements. Proceedings of the 2010
ACM-IEEE International Symposium on Empirical Software Engineering and Measurement ESEM'10, 2010, 1-10. https://doi.org/10.1145/1852786.1852810

17. Firesmith, D. Common Requirements Problems, their Negative Consequences, and the Industry Best Practices to Help Solve Them. Journal of Object Technology, 2007, 6(1), 17-33. https://doi.org/10.5381/ jot.2007.6.1.c2

18. Glinz, M. On Non-Functional Requirements. 15th IEEE International Requirements Engineering Conference (RE 2007), 2007, 21-26. https://doi.org/10.1109/ RE.2007.45

19. Groen, E. C., Schowalter, J., Kopczynska, S., Polst, S., Alvani, S. Is There Really a Need for Using NLP to Elicit Requirements? A Benchmarking Study to Assess Scalability of Manual Analysis. CEUR Workshop Proceedings, 2018.

20. Hindl, A., Ernst, N. A., Godfrey, M. W., Mylopoulo, J. Automated Topic Naming. Empirical Software Engineering, 2013, 18(6), 1125-1155. https://doi.org/10.1007/ s10664-012-9209-9

21. Hussain, A., Mkpojiog, E., Kama, F. The Role of Requirements in the Success or Failure of Software Projects. EJ Econjournals, 2016, 6(7S), 6-7\%.

22. Kang, J., Par, J. Generating a Linguistic Model for Requirement Quality Analysis. Proceedings of the 30th Pacific Asia Conference on Language, Information and Computation (PACLIC 30), Seoul, Republic of Korea, October 28-30, 2016, 439-447.

23. Kingma, D. P., Ba, J. Adam: A Method for Stochastic Optimization. 3rd International Conference for Learning Representations, 2015, 1-15.

24. Li, Y., Guzman, E., Tsiamoura, K., Schneide, F., Bruegge, B. Automated Requirements Extraction for Scientific Software. Procedia Computer Science, 2015, 51, 582591. https://doi.org/10.1016/j.procs.2015.05.326

25. Lu, M., Liang, P. Automatic Classification of Non-Functional Requirements from Augmented App User Reviews. Proceedings of the 21st International Conference on Evaluation and Assessment in Software Engineering EASE'17, 2017, 344-353. https://doi. org/10.1145/3084226.3084241

26. Mahmoud, A. An Information Theoretic Approach for Extracting and Tracing NonFunctional Requirements. 2015 IEEE 23rd International Requirements Engineering Conference (RE), 2015, 36-45. https://doi. org/10.1109/RE.2015.7320406 
27. Mahmoud, A., Williams, G. Detecting, Classifying, and Tracing NonFunctional Software Requirements. Requirements Engineering, 2016, 21(3), 357-381. https:// doi.org/10.1007/s00766-016-0252-8

28. Mairiza, D., Zowghi, D, Nurmulian, N. An Investigation into the Notion of NonFunctional Requirements. Proceedings of the 2010 ACM Symposium on Applied Computing - SAC'10, 2010, 311. https://doi. org/10.1145/17774088.17744153

29. Paixao, K. V. R., Felicio, C. Z., Delfim, F. M., Maia, M. D. A. On The Interplay between Non-Functional Requirements and Builds on Continuous Integration. IEEE International Working Conference on Mining Software Repositories, 2017, 479-482. https://doi.org/10.1109/ MSR.2017.33

30. Pedregosa, F., Varoquaux, G., Gramfort, A., Michel, V., Thirion, B., Grisel, O., Blondel, M., Prettenhofer, P., Weiss, R., Dubourg, V., Vanderplas, J., Passos, A., Cournapeau, D., Brucher, M., Perrot, M., Duchesnay, E. Scikit-learn: Machine Learning in Python. Journal of Machine Learning Research, 2011, 12, 2825-2830.

31. Portugal, R. L. Q., do Prado Leite, J. C. S. Extracting Requirements Patterns from Software Repositories. 2016 IEEE 24th International Requirements Engineering Conference Workshops (REW), 2016, 304-30\%. https:// doi.org/10.1109/REW.2016.056

32. Portugal, R. L. Q, Roque, H., do Prado Leite, J. C. S. A Corpus Builder: Retrieving Raw Data from Github for Knowledge Reuse in Requirements Elicitation. CEUR Workshop Proceedings, 2016, 48-54.

33. Rashwan, A., Ormandjieva, O., Witte, R. Ontology-Based Classification of Non-Functional Requirements in Software Specifications: A New Corpus and SVM-Based Classifier. 2013 IEEE 37th Annual Computer Software and Applications Conference, 2013, 381-386. https:// doi.org/10.1109/COMPSAC.2013.64

34. Sampaio, A., Loughran, N., Rashi, A., Rayson, P. Mining Aspects in Requirements. Early Aspects 2005: AspectOriented Requirements Engineering and Architecture Design Workshop, 2005.
35. Sawyer, P., Rayson, P., Garsid, R. REVERE: Support for Requirements Synthesis from Documents. Information Systems Frontiers, 2002, 4(3), 343-353. https://doi. org/10.1023/A:1019918908208

36. Sharma, V. S., Ramnani, R. R., Sengupta S. A Framework for Identifying and Analyzing Non-functional Requirements from Text. Proceedings of the 4th International Workshop on Twin Peaks of Requirements and Architecture, 2014, 1-8. https://doi. org/10.1145/2593861.2593862

37. Slankas, J., Williams, L. Automated Extraction of Non-Functional Requirements in Available Documentation. 2013 1st International Workshop on Natural Language Analysis in Software Engineering (NaturaLiSE), 2013, 916. https://doi.org/10.1109/NAturaLiSE.2013.6611715

38. Tóth, L., Vidác, L. Study of Various Classifiers for Identification and Classification of Non-Functional Requirements. Proceedings of the 18th International Conference on Computational Science and Its Applications (ICCSA 2018), 2018, 492-503. https://doi. org/10.1007/978-3-319-95174-4_39

39. Zou, J., Xu, L., Guo, W., Yan, M., Yang, D., Zhang, X. Which Non-functional Requirements Do Developers Focus On? An Empirical Study on Stack Overflow Using Topic Analysis. 2015 IEEE/ACM 12th Working Conference on Mining Software Repositories, 2015, 446-449. https://doi.org/10.1109/MSR.2015.60

40. Zo, J., Xu, L., Yang, M., Zhang, X., Yang, D. Towards Comprehending the Non-functional Requirements Through Developers Eyes. Information and Software Technology, 2017, 84(C), 19-32. https://doi.org/10.1016/j.infsof.2016.12.003

41. Zou, X., Settimi, R., ClelandHuang, J. Improving Automated Requirements Trace Retrieval: A Study of TermBased Enhancement Methods. Empirical Software Engineering, 2010, 15(2), 119-146. https://doi.org/10.1007/ s10664-009-9114-z 